PROCEEDINGS OF THE

AMERICAN MATHEMATICAL SOCIETY

Volume 138, Number 6, June 2010, Pages 1969-1978

S 0002-9939(10)10273-1

Article electronically published on February 11, 2010

\title{
2-SELMER GROUPS OF QUADRATIC TWISTS OF ELLIPTIC CURVES
}

\author{
GEORGE BOXER AND PETER DIAO
}

(Communicated by Ken Ono)

\begin{abstract}
In this paper we investigate families of quadratic twists of elliptic curves. Addressing a speculation of Ono, we identify a large class of elliptic curves for which the parities of the "algebraic parts" of the central values $L\left(E^{(d)} / \mathbb{D} Q, 1\right)$, as $d$ varies, have essentially the same multiplicative structure as the coefficients $a_{d}$ of $L(E / \mathbb{D} Q, s)$. We achieve this by controlling the 2Selmer rank (à la Mazur and Rubin) when the Tamagawa numbers do not already dictate the parity.
\end{abstract}

\section{INTRODUCTION}

Let $E / \mathbb{Q}$ be an elliptic curve and let $L(E / \mathbb{Q}, s)$ be its $L$-function. The famous conjecture of Birch and Swinnerton-Dyer predicts that

$$
L(E / \mathbb{Q}, 1)=\left\{\begin{array}{lll}
\frac{|\omega(E / \mathbb{Q})| \Omega_{E} \prod_{p} c_{p}}{\left|E_{\text {Tor }}\right|^{2}} & \text { if } & \operatorname{rank}(E / \mathbb{Q})=0 \\
0 & \text { if } & \operatorname{rank}(E / \mathbb{Q})>0,
\end{array}\right.
$$

where $|\amalg(E / \mathbb{Q})|$ denotes the order of the Shafarevich-Tate group (which we will temporarily assume is finite), $c_{p}$ denotes the Tamagawa number at $p, \Omega_{E}$ denotes the period of $E$, and $\left|E_{\text {Tor }}\right|$ is the size of the group of rational torsion points of $E$. For convenience we define:

$$
\begin{aligned}
L^{\operatorname{alg}}(E / \mathbb{Q}, 1) & :=\frac{L(E / \mathbb{Q}, 1)}{\Omega_{E}}, \\
L^{\mathrm{BSD}}(E / \mathbb{Q}, 1) & := \begin{cases}\frac{|\omega(E / \mathbb{Q})| \prod_{p} c_{p}}{\left|E_{\mathrm{Tor}}\right|^{2}} & \text { if } \operatorname{rank}(E / \mathbb{Q})=0 \\
0 & \text { if } \operatorname{rank}(E / \mathbb{Q})>0 .\end{cases}
\end{aligned}
$$

The Birch and Swinnerton-Dyer conjecture predicts that $L^{\text {alg }}=L^{\mathrm{BSD}}$. We consider elliptic curves over $\mathbb{Q}$ without $\mathbb{Q}$-rational 2-torsion and investigate the parity of these quantities (even if they aren't integers) in families of quadratic twists. If $E / \mathbb{Q}$ is an elliptic curve and $d \neq 1$ is a squarefree integer, then recall that the quadratic twist $E^{(d)} / \mathbb{Q}$ of $E$ by $d$ is the unique elliptic curve over $\mathbb{Q}$ that is not isomorphic to $E$ but becomes isomorphic to $E$ over $\mathbb{Q}(\sqrt{d})$. The question of how $\operatorname{rank}\left(E^{(d)} / \mathbb{Q}\right)$ varies with $d$ is an extremely important one and is the subject of many conjectures. Perhaps the most notable is Goldfeld's conjecture, which predicts that $E^{(d)} / \mathbb{Q}$ has rank 0 half the time and rank 1 half the time.

Received by the editors October 27, 2009.

2010 Mathematics Subject Classification. Primary 11G05, 11G40.

(C)2010 American Mathematical Society Reverts to public domain 28 years from publication 
This problem has been studied by Ono and Skinner [OS, O], who use Waldspurger's theory to study the nonvanishing of central values of $L$-functions by investigating the power of 2 dividing $L^{\text {alg }}$. More recently it has been studied in the work of Mazur and Rubin $\mathrm{MR}$ from the point of view of controlling 2-Selmer ranks, which can essentially be interpreted as studying the parity of $L^{\mathrm{BSD}}$. In principle, by combining these two works one can hope to prove the "Birch and Swinnerton-Dyer conjecture mod 2" for many elliptic curves in a family of quadratic twists.

Our work is motivated by the beautiful example of $E=X_{0}(11)$. Let

$$
f(z)=\sum_{n=1}^{\infty} a_{n} q^{n}=\eta(z)^{2} \eta(11 z)^{2}=q \prod_{n=1}^{\infty}\left(1-q^{n}\right)^{2}\left(1-q^{11 n}\right)^{2}
$$

be the weight 2 level 11 modular form associated to $X_{0}(11)$, where $q=e^{2 \pi i z}$. By the theory of Shimura, Waldspurger, and Kohnen, there is a weight $3 / 2$ modular form $F=\sum_{n=1}^{\infty} B_{n} q^{n}$ associated to $E$ whose Fourier coefficients encode the central $L$ values of negative quadratic twists of $E$. More precisely we have Kohnen's formula

$$
L\left(E^{(-d)} / \mathbb{Q}, 1\right)=\frac{\pi\langle f, f\rangle}{2\langle F, F\rangle} \frac{B_{D}^{2}}{\sqrt{D}}, \quad \text { for } \quad(d, 11)=1
$$

where $D=-\operatorname{disc}(\mathbb{Q}(\sqrt{-d}))$ and the $\langle\cdot, \cdot\rangle$ are the relevant Petersson inner products for $\Gamma_{0}(11)$ and $\Gamma_{0}(44)$. In this case, $F$ has the following nice description $\mathrm{Sh}$. We have that $B_{n}=\widehat{B}_{4 n}$, where $\widehat{B}_{n}$ is defined by

$$
\begin{aligned}
\widehat{F}(z) & =\sum_{n=1}^{\infty} \widehat{B}_{n} q^{n}=\theta(11 z) \eta(2 z) \eta(22 z) \\
& =\left(\sum_{n=-\infty}^{\infty} q^{11 n^{2}}\right)\left(q \prod_{n=1}^{\infty}\left(1-q^{2 n}\right)\left(1-q^{22 n}\right)\right) .
\end{aligned}
$$

Since $\widehat{F} \equiv f(\bmod 2)$, with a little work we see from Kohnen's formula (1.3) that for $d>0$ squarefree with $(d, 11)=1$, the parity of $L^{\text {alg }}\left(E^{(-d)} / \mathbb{Q}, 1\right)$ has a very nice description:

$$
L^{\operatorname{alg}}\left(E^{(-d)}, 1\right) \equiv a_{d} \quad(\bmod 2) .
$$

Using the arithmetic description of $f$, we have that for $d$ odd and squarefree, $a_{d}$ is odd if and only if for every prime $p$ dividing $d, E$ has no rational 2-torsion modulo $p$.

This leads to two natural questions:

(1) Can one directly prove (1.4) for $L^{\mathrm{BSD}}\left(E^{(-d)}, 1\right)$ ?

(2) Is this an example of a general phenomenon? In particular does the Birch and Swinnerton-Dyer conjecture predict (1.4) for a general class of elliptic curves?

We answer these questions for certain curves which we now define. We call an elliptic curve $E / \mathbb{Q}$ good if it satisfies all of the following:

(1) The 2-Selmer rank of $E$ is 0.

(2) The discriminant $\Delta$ of $E$ is negative.

(3) If $p$ is any prime for which $E$ has bad reduction, then $E$ has multiplicative reduction at $p$ and $v_{p}(\Delta)$ is odd.

(4) $E$ has good reduction at 2 and the reduction of $E \bmod 2$ has $j$-invariant 0. 
Remark. Condition (4) is equivalent to $E$ having a minimal model

$$
E: y^{2}+y=x^{3}+a_{2} x^{2}+a_{4} x+a_{6} .
$$

Furthermore note that this implies that the reduction of $E \bmod 2, \tilde{E}\left(\mathbb{F}_{2}\right)$, has no 2-torsion.

Remark. There are many good elliptic curves. For example, an elliptic curve is good if it has 2-Selmer rank $0, v_{2}(j)>0$, and discriminant that is negative, squarefree, and prime to 2 .

In the course of this paper, the following condition arises sufficiently often that we give it a name. If $E / \mathbb{Q}$ is an elliptic curve, we shall refer to a squarefree integer $d$ as 2-trivial for $E$ if $E$ has no rational 2-torsion $\bmod p$ for every odd prime $p \mid d$. For good $E$, such as $X_{0}(11)$, we prove the following theorem for 2-trivial integers.

Theorem 1.1. Let $E / \mathbb{Q}$ be a good elliptic curve. If $d$ is a squarefree 2-trivial integer with $(d, \Delta)=1$, then

$$
\operatorname{dim}_{\mathbb{F}_{2}}\left(\operatorname{Sel}_{2}\left(E^{(d)}\right)\right)= \begin{cases}0 & \text { if } d \text { is odd } \\ 1 & \text { if } d \text { is even } .\end{cases}
$$

In particular for such odd $d$ we have that $\operatorname{rank}\left(E^{(d)} / \mathbb{Q}\right)=0$.

Remark. Mazur and Rubin [MR, Prop. 4.2] show that for an arbitrary elliptic curve $E / \mathbb{Q}$ with 2-Selmer rank 0 , if $0<d \equiv 1(\bmod 8 \Delta)$ is a 2-trivial squarefree integer, then $E^{(d)} / \mathbb{Q}$ has 2-Selmer rank 0 as well. We show that with some assumptions on $E / \mathbb{Q}$, we can control the 2-Selmer rank of $E^{(d)} / \mathbb{Q}$ for all 2-trivial squarefree integers $d$ prime to $\Delta$.

Remark. If we consider the set $C_{r}(X)$ of squarefree integers $|d|<X$ with $r$ prime factors, as considered in $[\mathrm{OS}$, then the Chebotarev density theorem implies that for an elliptic curve $E$ with no rational 2-torsion,

$$
\#\left\{d \in C_{r}(X) \mid d \text { is 2-trivial for } E\right\} \gg_{r}\left|C_{r}(X)\right| .
$$

Since the implied constants tend to 0 as $r \rightarrow+\infty$, this result falls short of proving that a positive proportion of quadratic twists have rank 0 . On the other hand we still have

$$
\#\{0<d<X \mid d \text { is squarefree and 2-trivial for } E\} \gg \frac{X}{(\log X)^{1-\alpha}}
$$

for some $\alpha>0$.

It is important to relate Theorem 1.1 to the parity of $L^{\mathrm{BSD}}\left(E^{(d)}, 1\right)$ for all $d$ that are squarefree (positive or negative).

Theorem 1.2. Let $E / \mathbb{Q}$ be a good elliptic curve, and let $a_{n}$ be defined by

$$
L(E / \mathbb{Q}, s)=\sum_{n=1}^{\infty} \frac{a_{n}}{n^{s}}
$$

If $d$ is a squarefree integer, then

$$
\begin{cases}L^{\mathrm{BSD}}\left(E^{(d)}, 1\right) \equiv a_{|d|} \quad(\bmod 2) & \text { if }(d, \Delta)=1 \\ L^{\mathrm{BSD}}\left(E^{(d)}, 1\right) \equiv 0 \quad(\bmod 2) & \text { if }(d, \Delta)>1\end{cases}
$$


Remark. Strictly speaking, the statement of this theorem presumes the conjectural finiteness of $|\amalg(E / \mathbb{Q})|$. However, we can define the parity of $|\amalg(E / \mathbb{Q})|$ in order to avoid this issue. We say that $|\amalg(E / \mathbb{Q})|$ has odd order if and only if its 2-part is trivial. Thus when suitably interpreted, this theorem can be stated unconditionally.

This paper is structured as follows. In Section 2 we recall the work of Mazur and Rubin and derive conditions which imply the conclusion of Theorem 1.1. We carry out calculations in Section 3 to obtain Theorem 1.1. The deduction of Theorem 1.2 from Theorem 1.1 amounts to calculating Tamagawa numbers, which is done in Section 4

\section{Preliminaries}

We now recall the recent work of Mazur and Rubin $\mathrm{MR}$ which allows us to prove the following key criterion.

Theorem 2.1. Let $E / \mathbb{Q}$ be a good elliptic curve. If $d$ is a squarefree 2-trivial integer with $(d, \Delta)=1$, then the following are true:

(1) If $d \equiv 1(\bmod 4)$, then $\operatorname{dim}_{\mathbb{F}_{2}}\left(\operatorname{Sel}_{2}\left(E^{(d)}\right)\right)=0$.

(2) Otherwise, we have

$$
\begin{aligned}
& \quad \operatorname{dim}_{\mathbb{F}_{2}}\left(\operatorname{Sel}_{2}\left(E^{(d)} / \mathbb{Q}\right)\right) \leq \operatorname{dim}_{\mathbb{F}_{2}}\left(E\left(\mathbb{Q}_{2}\right) / 2 E\left(\mathbb{Q}_{2}\right)\right) \\
& \text { and } \\
& \operatorname{dim}_{\mathbb{F}_{2}}\left(\operatorname{Sel}_{2}\left(E^{(d)} / \mathbb{Q}\right)\right) \equiv \operatorname{dim}_{\mathbb{F}_{2}}\left(E\left(\mathbb{Q}_{2}\right) / N_{\mathbb{Q}_{2}}^{\mathbb{Q}_{2}(\sqrt{d})} E\left(\mathbb{Q}_{2}(\sqrt{d})\right)\right) \quad(\bmod 2) .
\end{aligned}
$$

2.1. Mazur and Rubin's results revisited. The problem of calculating MordellWeil groups reduces to calculating orders of 2-Selmer groups and 2-torsion in the Shafarevich-Tate group. Under the standard 2-descent this is easiest when $E$ has full rational 2-torsion. The approach of Mazur and Rubin for studying the 2-Selmer rank of quadratic twists is more versatile as it works without any assumption on the 2-torsion.

We restrict our attention to $\mathbb{Q}$ even though most of this discussion holds for a general number field. Let $v$ be a place of $\mathbb{Q}$. There is a short exact sequence of $\operatorname{Gal}\left(\overline{\mathbb{Q}_{v}} / \mathbb{Q}_{v}\right)$-modules

$$
0 \rightarrow E\left(\overline{\mathbb{Q}_{v}}\right)[2] \rightarrow E\left(\overline{\mathbb{Q}_{v}}\right) \stackrel{2}{\rightarrow} E\left(\overline{\mathbb{Q}_{v}}\right) \rightarrow 0 .
$$

Taking Galois cohomology one obtains the Kummer map $\delta$ :

$$
0 \rightarrow E\left(\mathbb{Q}_{v}\right) / 2 E\left(\mathbb{Q}_{v}\right) \stackrel{\delta}{\rightarrow} H^{1}\left(\mathbb{Q}_{v}, E[2]\right) .
$$

Following $[\mathrm{MR}]$ we denote the image of the Kummer map by $H_{f}^{1}\left(\mathbb{Q}_{v}, E[2]\right)$. For each $v$ there is a localization map

$$
\operatorname{loc}_{v}: H^{1}(\mathbb{Q}, E[2]) \rightarrow H^{1}\left(\mathbb{Q}_{v}, E[2]\right),
$$

and the 2-Selmer group $\operatorname{Sel}_{2}(E / \mathbb{Q})$ is defined to be the subgroup of $H^{1}(\mathbb{Q}, E[2])$ of elements whose images under $\operatorname{loc}_{v}$ are in $H_{f}^{1}\left(\mathbb{Q}_{v}, E[2]\right)$ for all $v$.

Now let $E^{(d)}$ be a quadratic twist of $E$. There is a natural isomorphism of $\operatorname{Gal}(\overline{\mathbb{Q}} / \mathbb{Q})$-modules, $E[2] \cong E^{(d)}[2]$. This suggests a natural way of studying how the 2-Selmer group varies in quadratic twists, namely: we can view $\operatorname{Sel}_{2}(E / \mathbb{Q})$ and $\operatorname{Sel}_{2}\left(E^{(d)} / \mathbb{Q}\right)$ as living in the same space $H^{1}(\mathbb{Q}, E[2])$ but being cut out by different local conditions. Thus the question of how the 2-Selmer group varies in families 
of quadratic twists can be reduced to studying how these local conditions change. This is the point of view taken in MR.

In fact, if $E$ and $E^{(d)}$ are as above, then there is a finite set of places $T$ of $\mathbb{Q}$, depending on $d$ (see Proposition 2.4 below), such that for all places $v$ not in $T$,

$$
H_{f}^{1}\left(\mathbb{Q}_{v}, E[2]\right)=H_{f}^{1}\left(\mathbb{Q}_{v}, E^{(d)}[2]\right) .
$$

Now following $\left[\mathrm{MR}\right.$, Def. 3.1] we define two additional subgroups of $H^{1}(\mathbb{Q}, E[2])$ cut out by local conditions. We define the relaxed 2-Selmer group $\mathcal{S}^{T}$ to be the subgroup of $H^{1}(\mathbb{Q}, E[2])$ of elements whose images under $\operatorname{loc}_{v}$ are in $H_{f}^{1}\left(\mathbb{Q}_{v}, E[2]\right)$ for all places $v$ outside of $T$. Additionally we define the strict 2-Selmer group $\mathcal{S}_{T}$ to be the subgroup of $\mathcal{S}^{T}$ of elements which additionally are killed by $\operatorname{loc}_{v}$ for all places $v$ in $T$. More succinctly there are exact sequences

$$
\begin{aligned}
0 & \rightarrow \mathcal{S}^{T} \rightarrow H^{1}(\mathbb{Q}, E[2]) \rightarrow \bigoplus_{v \notin T} H^{1}\left(\mathbb{Q}_{v}, E[2]\right) / H_{f}^{1}\left(\mathbb{Q}_{v}, E[2]\right), \\
0 & \rightarrow \mathcal{S}_{T} \rightarrow \mathcal{S}^{T} \rightarrow \bigoplus_{v \in T} H^{1}\left(\mathbb{Q}_{v}, E[2]\right)
\end{aligned}
$$

By definition we have

$$
\mathcal{S}_{T} \subset \operatorname{Sel}_{2}(\mathbb{E} / \mathbb{Q}) \subset \mathcal{S}^{T} \quad \text { and } \quad \mathcal{S}_{T} \subset \operatorname{Sel}_{2}\left(\mathbb{E}^{(d)} / \mathbb{Q}\right) \subset \mathcal{S}^{T} .
$$

In light of this the following result bounds the 2-Selmer rank of $E^{(d)}$.

Proposition 2.2 ([MR, Lemma 3.2]). If $T, \mathcal{S}_{T}$, and $\mathcal{S}^{T}$ are as above, then

$$
\operatorname{dim}_{\mathbb{F}_{2}}\left(\mathcal{S}^{T} / \mathcal{S}_{T}\right)=\sum_{v \in T} \operatorname{dim}_{\mathbb{F}_{2}}\left(H_{f}^{1}\left(K_{v}, E[2]\right)\right) .
$$

Then the following congruence of Kramer provides a "local formula" for comparing the parity of the 2-Selmer rank of a curve $E$ and that of its quadratic twist $E^{(d)}$.

Theorem 2.3 (Kramer $\underline{\mathrm{Kr}}$ ). We have that

$$
\operatorname{dim}_{\mathbb{F}_{2}}\left(\operatorname{Sel}_{2}\left(E^{(d)} / \mathbb{Q}\right)\right) \equiv \operatorname{dim}_{\mathbb{F}_{2}}\left(\operatorname{Sel}_{2}(E / \mathbb{Q})\right)+\sum_{v} \delta_{v}(E, d) \quad(\bmod 2),
$$

where the sum is taken over all places of $\mathbb{Q}$ and the local factor $\delta_{v}(E, d)$ is defined by

$$
\delta_{v}(E, d)=\operatorname{dim}_{\mathbb{F}_{2}}\left(E\left(\mathbb{Q}_{v}\right) / N_{\mathbb{Q}_{v}}^{\mathbb{Q}_{v}(\sqrt{d})} E\left(\mathbb{Q}_{v}(\sqrt{d})\right)\right) .
$$

In view of these results, we recall the following criteria for the equality of $H_{f}^{1}\left(\mathbb{Q}_{v}, E[2]\right)$ and $H_{f}^{1}\left(\mathbb{Q}_{v}, E^{(d)}[2]\right)$ and the triviality of $\delta_{v}(E, d)$.

Proposition 2.4 ([MR, Lemma 2.5]). Let $E^{(d)}$ be a quadratic twist of $E / \mathbb{Q}$ and let $F=\mathbb{Q}(\sqrt{d})$ be the corresponding quadratic extension. Let $v$ be a place of $\mathbb{Q}$ satisfying at least one of the following conditions:

(1) $v$ is a finite place where $E$ has good reduction and $F / \mathbb{Q}$ is unramified.

(2) $v$ is a finite place where $E$ has multiplicative reduction and $\operatorname{ord}_{v}\left(\Delta_{E}\right)$ is odd, and $F / \mathbb{Q}$ is unramified at $v$.

(3) $v$ is the real place and $\Delta_{E}<0$.

(4) $v$ is a finite place other than 2 and $E\left(\mathbb{Q}_{v}\right)[2]=0$.

Then $H_{f}^{1}\left(\mathbb{Q}_{v}, E[2]\right)=H_{f}^{1}\left(\mathbb{Q}_{v}, E^{(d)}[2]\right)$ and $\delta_{v}(E, d)=0$. 
2.2. Proof of Theorem 2.1. We claim that for all places $v$ of $\mathbb{Q}$ other than the prime 2 , we have that $H_{f}^{1}\left(\mathbb{Q}_{v}, E[2]\right)=H_{f}^{1}\left(\mathbb{Q}_{v}, E^{(d)}[2]\right)$ and $\delta_{v}(E, d)=0$. We consider three cases. If $v$ is the real place, then this follows from the fact that $\Delta<0$ and case (3) of Proposition 2.4. If $v$ is a finite place of multiplicative reduction, then by assumption $\operatorname{ord}_{v}(\Delta)$ is odd and $\mathbb{Q}(\sqrt{d})$ is unramified at $v($ since $(d, \Delta)=1$ ), and so the claim follows from case (2) of Proposition 2.4. Now assume that $v \neq 2$ is a finite place of good reduction, corresponding to the prime $p$. If $v$ is unramified in $\mathbb{Q}(\sqrt{d})$, then the claim follows from case (1) of Proposition 2.4. Finally if $v$ ramifies in $\mathbb{Q}(\sqrt{d})$, then $p$ divides $d$ and so by assumption $E$ has no 2-torsion mod $p$ and thus the claim follows from case (4) of Proposition 2.4.

To prove the first statement of the theorem, observe that if $d \equiv 1(\bmod 4)$, then 2 does not ramify in $\mathbb{Q}(\sqrt{d})$, and since $E$ has good reduction at 2, Proposition 2.4 implies that $H_{f}^{1}\left(\mathbb{Q}_{2}, E[2]\right)=H_{f}^{1}\left(\mathbb{Q}_{2}, E^{(d)}[2]\right)$. Thus the local conditions defining $\operatorname{Sel}_{2}(E / \mathbb{Q})$ and $\operatorname{Sel}_{2}\left(E^{(d)} / \mathbb{Q}\right)$ agree, so $\operatorname{dim}_{\mathbb{F}_{2}}\left(\operatorname{Sel}_{2}\left(E^{(d)} / \mathbb{Q}\right)\right)=0$.

Now we prove the second statement. Note that (2.2) follows immediately from Theorem 2.3 and the fact that $\operatorname{dim}_{\mathbb{F}_{2}}\left(\operatorname{Sel}_{2}(E / \mathbb{Q})\right)=0$. As for (2.1), the local conditions defining $\operatorname{Sel}_{2}(E / \mathbb{Q})$ and $\operatorname{Sel}_{2}\left(E^{(d)} / \mathbb{Q}\right)$ agree at all places except $v=2$. Thus with the notation as above, we can take $T=\{2\}$ and consider the corresponding strict and relaxed 2-Selmer groups. Since $\mathcal{S}_{T} \subset \operatorname{Sel}_{2}(E / \mathbb{Q})=0$, Proposition 2.2 implies that

$$
\operatorname{dim}_{\mathbb{F}_{2}}\left(\mathcal{S}^{T} / \mathcal{S}_{T}\right)=\operatorname{dim}_{\mathbb{F}_{2}}\left(\mathcal{S}^{T}\right)=\operatorname{dim}_{\mathbb{F}_{2}}\left(E\left(\mathbb{Q}_{2}\right) / 2 E\left(\mathbb{Q}_{2}\right)\right) .
$$

Since $\operatorname{Sel}_{2}\left(E^{(d)} / \mathbb{Q}\right) \subset \mathcal{S}^{T}$, the result follows.

\section{Proof of Theorem 1.1}

In view of Theorem 2.1, we see that the proof of Theorem 1.1 is reduced to the calculation of the dimension of $E\left(\mathbb{Q}_{2}\right) / 2 E\left(\mathbb{Q}_{2}\right)$ and the local factors $\delta_{2}(E, d)$. In this direction we first prove the following:

Proposition 3.1. Let $E / \mathbb{Q}_{2}$ be an elliptic curve with good reduction at 2 and with $E \bmod 2$ having $j$-invariant 0 . Then we have

$$
\operatorname{dim}_{\mathbb{F}_{2}}\left(E\left(\mathbb{Q}_{2}\right) / 2 E\left(\mathbb{Q}_{2}\right)\right)=1 \text {. }
$$

Proof. Let $E$ have a minimal model

$$
E: y^{2}+y=x^{3}+a_{2} x^{2}+a_{4} x+a_{6} .
$$

We recall a few standard facts about elliptic curves over local fields (see [S1, p. 174]). There is an exact sequence

$$
0 \rightarrow E_{1}\left(\mathbb{Q}_{2}\right) \rightarrow E\left(\mathbb{Q}_{2}\right) \rightarrow \tilde{E}\left(\mathbb{F}_{2}\right) \rightarrow 0,
$$

where $\tilde{E}$ is the reduction of $E \bmod 2$. Since $\tilde{E}\left(\mathbb{F}_{2}\right)$ is finite and has order prime to 2 , there is an isomorphism

$$
E\left(\mathbb{Q}_{2}\right) / 2 E\left(\mathbb{Q}_{2}\right) \cong E_{1}\left(\mathbb{Q}_{2}\right) / 2 E_{1}\left(\mathbb{Q}_{2}\right)
$$

The group $E_{1}\left(\mathbb{Q}_{2}\right)$ has a description in terms of the formal group of $E$. More precisely, there is a formal power series with coefficients in $\mathbb{Z}_{2}$,

$$
F\left(z_{1}, z_{2}\right)=z_{1}+z_{2}-a_{1} z_{1} z_{2}-a_{2}\left(z_{1}^{2} z_{2}+z_{1} z_{2}^{2}\right)+\cdots
$$


that endows the set $2 \mathbb{Z}_{2}$ with a group structure $\mathcal{F}\left(2 \mathbb{Z}_{2}\right)$. Then there is an isomorphism of groups $E_{1}\left(\mathbb{Q}_{2}\right) \cong \mathcal{F}\left(2 \mathbb{Z}_{2}\right)$. Furthermore by [S1, p. 126] the subgroup $\mathcal{F}\left(4 \mathbb{Z}_{2}\right)$ is isomorphic to $4 \mathbb{Z}_{2}$ under addition. In particular this implies that $2 \mathcal{F}\left(4 \mathbb{Z}_{2}\right)=\mathcal{F}\left(8 \mathbb{Z}_{2}\right)$. Now given an arbitrary element $c_{1} 2+d$ of $2 \mathbb{Z}_{2}$, with $c_{1} \in\{0,1\}$ and $d \in 4 \mathbb{Z}_{2}$, we have by the formal group law that

$$
2 \cdot\left(c_{1} 2+d\right)=4 c_{1}+O\left(2^{3}\right) .
$$

Thus $2 \mathcal{F}\left(2 \mathbb{Z}_{2}\right)=\mathcal{F}\left(4 \mathbb{Z}_{2}\right)$ and the result follows.

Remark. The assumption that the reduction of $E \bmod 2$ has $j$-invariant 0 is essential here. If $E / \mathbb{Q}_{2}$ had good reduction and the reduction $\bmod 2$ had $j$-invariant 1, then we would have $\operatorname{dim}_{\mathbb{F}_{2}}\left(E\left(\mathbb{Q}_{2}\right) / 2 E\left(\mathbb{Q}_{2}\right)\right)>1$.

Remark. An alternative way to prove Proposition 3.1 is to use the standard fact that $E\left(\mathbb{Q}_{2}\right) \cong \mathbb{Z}_{2} \times E\left(\mathbb{Q}_{2}\right)_{\text {Tor }}$ and then observe that the assumptions of the proposition imply that $E\left(\mathbb{Q}_{2}\right)$ has no 2-torsion by [Se, Prop. 12].

Turning to the local $\delta$ factors we have the following.

Proposition 3.2. Let $E / \mathbb{Q}_{2}$ be an elliptic curve with good reduction at 2 and with $E$ mod 2 having $j$-invariant 0 . If $K / \mathbb{Q}_{2}$ is a ramified quadratic extension, then

$$
\operatorname{dim}_{\mathbb{F}_{2}}\left(E\left(\mathbb{Q}_{2}\right) / N_{\mathbb{Q}_{2}}^{K} E(K)\right)= \begin{cases}0 & \text { if } K=\mathbb{Q}_{2}(\sqrt{d}) \text { with } d=3,7 \\ 1 & \text { if } K=\mathbb{Q}_{2}(\sqrt{d}) \text { with } d=2,6,10,14 .\end{cases}
$$

Proof. Let $E$ have a minimal model

$$
E: y^{2}+y=x^{3}+a_{2} x^{2}+a_{4} x+a_{6} .
$$

For brevity we denote $N_{\mathbb{Q}_{2}}^{K}$ by $N$. We have a commutative diagram

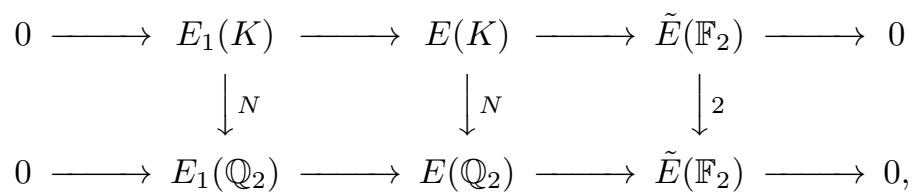

from which we obtain an isomorphism $E\left(\mathbb{Q}_{2}\right) / N E(K) \cong E_{1}\left(\mathbb{Q}_{2}\right) / N E_{1}(K)$. Let $\pi$ denote a uniformizer for $K$ and let $\mathcal{O}_{K}$ denote its ring of integers. Then again by [S1, p. 126] we have isomorphisms $\mathcal{F}\left(\pi^{3} \mathcal{O}_{K}\right) \cong \pi^{3} \mathcal{O}_{K}$ and $\mathcal{F}\left(4 \mathbb{Z}_{2}\right) \cong 4 \mathbb{Z}_{2}$. Furthermore these isomorphisms are given by the same convergent power series with coefficients in $\mathbb{Q}_{2}$. Thus there is a commutative diagram

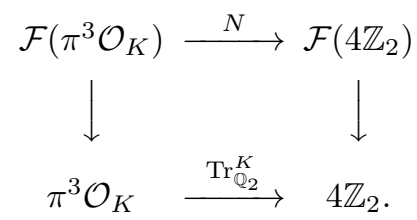

Since $\operatorname{Tr} \pi^{3} \mathcal{O}_{K}=8 \mathbb{Z}_{2}$, we have $\mathcal{F}\left(8 \mathbb{Z}_{2}\right) \subset N \mathcal{F}\left(\pi \mathcal{O}_{K}\right)$. Now consider the element $2 \in \mathcal{F}\left(\pi \mathcal{O}_{K}\right)$. Using the formal group law we compute its norm

$$
N(2)=F(2,2)=4-O\left(2^{3}\right) .
$$

Thus $N \mathcal{F}\left(\pi^{2} \mathcal{O}_{K}\right)=\mathcal{F}\left(4 \mathbb{Z}_{2}\right)$. 
To complete the proposition it remains to compute $N(\pi)$. Now we split into two cases. First suppose $K=\mathbb{Q}_{2}(\sqrt{d})$ with $d=3$ or 7 . Then we can take $\pi=1+\sqrt{d}$. By the formal group law we have

$$
N(\pi)=F(\pi, \bar{\pi})=\pi+\bar{\pi}+O\left(\pi^{3}\right)=2+O\left(2^{2}\right) .
$$

Thus in this case $N \mathcal{F}\left(\pi \mathcal{O}_{K}\right)=\mathcal{F}\left(2 \mathbb{Z}_{2}\right)$ as required. Now if we have $K=\mathbb{Q}_{2}(\sqrt{d})$ with $d=2,6,10$, or 14 we can take $\pi=\sqrt{d}$ and we have

$$
N(\pi)=F(\pi, \bar{\pi})=\pi+\bar{\pi}+O\left(\pi^{3}\right)=0+O\left(2^{2}\right) .
$$

Thus in this case $N \mathcal{F}\left(\pi \mathcal{O}_{K}\right)=\mathcal{F}\left(4 \mathbb{Z}_{2}\right)$, which has index 2 in $\mathcal{F}\left(2 \mathbb{Z}_{2}\right)$, completing the proof.

Proof of Theorem 1.1. Let $d$ prime to $\Delta$ be a squarefree, 2-trivial integer for $E$. If $d \equiv 1(\bmod 4)$, then the result is immediate from the first part of Theorem 2.1. Otherwise, the second part of Theorem 2.1 implies that $\operatorname{dim}_{\mathbb{F}_{2}}\left(\operatorname{Sel}_{2}\left(E^{(d)}\right)\right) \leq$ $\operatorname{dim}_{\mathbb{F}_{2}} E\left(\mathbb{Q}_{2}\right) / 2 E\left(\mathbb{Q}_{2}\right)=1$ with the second equality following from Proposition 3.1. Thus $\operatorname{dim}_{\mathbb{F}_{2}}\left(\operatorname{Sel}_{2}\left(E^{(d)}\right)\right)$ is determined by its parity, which is computed as a result of Theorem 2.1 and Proposition 3.2 .

\section{Proof of Theorem 1.2}

The proof of Theorem 1.2 uses the following calculation of Tamagawa numbers for quadratic twists.

Proposition 4.1. Let $E / \mathbb{Q}$ be a good elliptic curve and let d be a squarefree integer. The following are true:

(1) If $(d, \Delta)=1$, then the Tamagawa factor $\prod_{p} c_{p}\left(E^{(d)}\right)$ is odd if and only if $d$ is 2-trivial for $E$.

(2) If $(d, \Delta)>1$, then the Tamagawa factor $\prod_{p} c_{p}\left(E^{(d)}\right)$ is even.

Proof. First we prove (1). If $p$ is a prime of bad reduction, then by assumption $p$ is odd; also $E$ has multiplicative reduction at $p$ and $v_{p}\left(\Delta_{E}\right)$ is odd. Then since $(d, \Delta)=1, E^{(d)}$ retains multiplicative reduction at $p$ and $v_{p}\left(\Delta_{E}\right)=v_{p}\left(\Delta_{E^{(d)}}\right)$, so the Tamagawa factor $c_{p}\left(\Delta_{E^{(d)}}\right)$ is odd (it is either 1 or $v_{p}\left(\Delta_{E}\right)$ depending on whether $E^{(d)}$ has split or non-split multiplicative reduction at $p$ [S2, p. 366]).

Next we consider the prime 2 . We claim that $c_{2}$ is always 1 . By assumption, $E$ has a minimal Weierstrass model of the form

$$
E: y^{2}+y=x^{3}+a_{2} x^{2}+a_{4} x+a_{6} .
$$

First suppose $d$ is even and make the change of variables

$$
x=\frac{x^{\prime}}{d}, \quad y=\frac{y^{\prime}}{d^{3 / 2}}-\frac{1}{2} .
$$

This yields a Weierstrass equation for $E^{(d)}$,

$$
E^{(d)}: y^{2}=x^{3}+a_{2}^{\prime} x^{2}+a_{4}^{\prime} x+a_{6}^{\prime},
$$

with coefficients

$$
\begin{aligned}
& a_{2}^{\prime}=d a_{2}, \\
& a_{4}^{\prime}=d^{2} a_{4}, \\
& a_{6}^{\prime}=d^{3}\left(a_{6}+\frac{1}{4}\right) .
\end{aligned}
$$


Since $d$ is even, the $a_{i}^{\prime}$ are integers and we apply Tate's algorithm. Since $a_{6}^{\prime} \equiv 2$ (mod 4) the algorithm terminates at step 3 of [S2, p. 366] and we see that $E^{(d)}$ has Kodaira symbol II at 2 and $c_{2}=1$.

Now suppose $d$ is odd. This time make the change of variables

$$
x=\frac{x^{\prime}}{4 d}, \quad y=\frac{y^{\prime}}{8 d^{3 / 2}}+\frac{1}{2 d^{3 / 2}}-\frac{1}{2}
$$

to obtain a Weierstrass equation for $E^{(d)}$ of the form

$$
E^{(d)}: y^{2}+8 y=x^{3}+a_{2}^{\prime} x^{2}+a_{4}^{\prime} x+a_{6}^{\prime}
$$

with coefficients

$$
\begin{aligned}
& a_{2}^{\prime}=4 d a_{2}, \\
& a_{4}^{\prime}=16 d^{2} a_{4}, \\
& a_{6}^{\prime}=64 d^{3} a_{6}+16\left(d^{3}-1\right) .
\end{aligned}
$$

If $d \equiv 1(\bmod 4)$, then $64 \mid a_{6}^{\prime}$ and we can make the change of variables $x=4 x^{\prime}$ and $y=8 y^{\prime}$ to see that $E^{(d)}$ has good reduction at 2 and thus $c_{2}=1$. On the other hand if $d \equiv 3(\bmod 4)$, then 32 is the largest power of 2 dividing $a_{6}^{\prime}$, and following Tate's algorithm we end up in step 10 of [S2, p. 368]; thus $E^{(d)}$ has Kodaira symbol II ${ }^{*}$ at 2 and $c_{2}=1$.

If $p$ is an odd prime of good reduction and $(p, d)=1$, then $E^{(d)}$ has good reduction at $p$ and so $c_{p}=1$. If $p$ divides $d$, then we can easily compute $c_{p}$ using Tate's algorithm. Since $p$ is odd, $E$ has a model which is minimal at $p$ of the form $y^{2}=f(x)$. Then the quadratic twist of $E$ by $d$ is given by the equation $E^{(d)}: y^{2}=d^{3} f(x / d)$. Following Tate's algorithm we end up in step 6 of [S2, p. 367], and it tells us that $E^{(d)}$ has Kodaira symbol $\mathrm{I}_{0}^{*}$ at $p$ and that $c_{p}$ is equal to 1 plus the number of roots of the polynomial $\left(d^{\prime}\right)^{3} f\left(x / d^{\prime}\right)$ where $d^{\prime}=d / p$. But this is just the number of 2-torsion of $E^{\left(d^{\prime}\right)} \bmod p$, which is the same as the number of 2 -torsion of $E \bmod p$. The claim follows.

Now we prove (2). Take any $p \mid(\Delta, d)$. Then $E$ has multiplicative reduction at $p$. Let $n=v_{p}(\Delta)$. Then by $\left[\mathbf{S 2}\right.$, p. 365], $E^{(d)}$ has Kodaira symbol $I_{n}^{*}$ at $p$, and by [S2, p. 367], $c_{p}=2$ or 4 . Thus the Tamagawa product for $E^{(d)}$ is even as claimed.

Proof of Theorem 1.2. First we characterize the parity of $a_{|d|}$ for $d$ squarefree. Since $E$ is assumed to have good reduction at 2 and has no 2-torsion modulo $2, a_{2}$ is even. Thus for $d$ even, $a_{|d|}$ is even by multiplicativity. On the other hand if $d$ is odd, then $a_{|d|}$ is odd if and only if $d$ is 2-trivial for $E$.

Now we consider $L^{\mathrm{BSD}}\left(E^{(d)}, 1\right)$. In the case where $(d, \Delta)>1$, either $\operatorname{rank}\left(E^{(d)}\right)>$ 0 or $\operatorname{rank}\left(E^{(d)}\right)=0$ and part (2) of Proposition 4.1 implies that the Tamagawa factor for $E^{(d)}$ is even. Either way $L^{\mathrm{BSD}}\left(E^{(d)}, 1\right)$ is even.

Now assume $(d, \Delta)=1$. We split into two cases. First suppose $d$ is not 2trivial. Then either $\operatorname{rank}\left(E^{(d)}\right)>0$ and $L^{\mathrm{BSD}}\left(E^{(d)}, 1\right)=0$ or $\operatorname{rank}\left(E^{(d)}\right)=0$ and $L^{\mathrm{BSD}}\left(E^{(d)}, 1\right)$ is still even as the Tamagawa product is even by Proposition 4.1 .

Now suppose $d$ is 2-trivial. If $d$ is odd, then by Theorem $1.1 E^{(d)}$ has 2-Selmer rank 0. Thus $\operatorname{rank}\left(E^{(d)}\right)=0$ and $\left|\amalg\left(E^{(d)}\right)\right|$ is odd. Furthermore the Tamagawa product is odd by Proposition 4.1 and so $L^{\mathrm{BSD}}\left(E^{(d)}, 1\right)$ is odd in this case. Finally if $d$ is even, then by Theorem $1.1 E^{(d)}$ has 2-Selmer rank 1 . Thus either the rank 
of $E^{(d)}$ is 1 or $\left|\amalg\left(E^{(d)}\right)\right|$ is even, and either way $L^{\mathrm{BSD}}\left(E^{(d)}, 1\right)$ is even (of course conjecturally only the first situation can occur).

\section{ACKNOWLEDGMENTS}

The authors would like to thank Ken Ono for suggesting this problem and for many helpful discussions. The authors would also like to thank the referee for comments and pointing out an alternative proof of Proposition 3.1 .

\section{REFERENCES}

[Kr] K. Kramer, Arithmetic of elliptic curves upon quadratic extensions, Trans. Amer. Math. Soc. 264 (1981) 121-135. MR597871 (82g:14028)

[MR] B. Mazur and K. Rubin, Ranks of twists of elliptic curves and Hilbert's tenth problem, preprint, available at http://arxiv.org/0904.3709

[O] K. Ono, Nonvanishing of quadratic twists of modular L-functions with applications for elliptic curves, J. Reine Angew. Math. 533 (2001) 81-97. MR1823865 (2002a:11051)

[OS] K. Ono and C. Skinner, Non-vanishing of quadratic twists of modular L-functions, Invent. Math. 134 (1998) 651-660. MR1660945 (2000a:11077)

[Se] J.-P. Serre, Propriétés galoisiennes des points d'ordre fini des courbes elliptiques, Invent. Math. 15 (1972) 259-331. MR0387283 (52:8126)

[Sh] T. Shintani, On construction of holomorphic cusp forms of half integral weight, Nagoya Math. J. 58 (1975) 83-126. MR0389772 (52:10603)

[S1] J.H. Silverman, The Arithmetic of Elliptic Curves, Graduate Texts in Mathematics, 106, New York: Springer-Verlag (1986). MR 817210 (87g:11070)

[S2] J.H. Silverman, Advanced Topics in the Arithmetic of Elliptic Curves, Graduate Texts in Mathematics, 151, New York: Springer-Verlag (1994). MR.1312368 (96b:11074)

Department of Mathematics, Mailbox 2704, Frist Center, Princeton University, Princeton, New Jersey 08544

E-mail address: gboxer@princeton.edu

Department of Mathematics, Mailbox 2704, Frist Center, Princeton University, Princeton, New Jersey 08544

E-mail address: pdiao@princeton.edu 